remaining $4 \%$ bone series study) and $44 \%$ CT. In $61 \%$, the fracture was located in dorsal spine, in $28 \%$ in lumbar spine and the remaining $11 \%$ in the dorsal and lumbar spine. Previously, 44\% were diagnosed with osteoporosis and $38 \%$ were undergoing specific treatment (oral bisphosphonate: $23 \%$, bisphosphonate iv: $20 \%$, denosumab: $20 \%$, SERM: $8 \%$, PTH: $6 \%$ and only calcium +vitamin supplements D: $23 \%$ )

Three months after the identification of the VF, $66 \%$ did not receive specific treatment for osteoporosis, $11 \%$ were referred to Rheumatology (initiating treatment in all) and $3 \%$ to Traumatology. Three (3\%) of the patients had died and $2(2 \%)$ had moved away.

Conclusions: Despite being reflected in the radiological report, a significant number of patients with vertebral fracture do not receive antiosteoporotic treatment, 3 months later.

Acknowledgements: The study was supported with a research grant from the Association for Research in Rheumatology of Marina Baixa (AIRE-MB).

Disclosure of Interest: None declared

DOI: 10.1136/annrheumdis-2018-eular.4958

\section{THU0480 MULTI-DISCIPLINARY FRACTURE LIAISON SERVICE IN THE NORTH AREA OF GRAN CANARIA; 6 YEARS EXPERIENCE}

A. Naranjo ${ }^{1}$, S. Ojeda ${ }^{1}$, S. Rodríguez ${ }^{2}$, A. Saavedra ${ }^{1}$, F. Santana ${ }^{1}$, A. Molina ${ }^{1}$ A. Gonzalez ${ }^{3}$, N. Martin ${ }^{4}$, M. Afonso ${ }^{5}$, C. Dominguez ${ }^{5}$, I. Beirutti ${ }^{6}, \mathrm{O}$. Suarez ${ }^{7}$, T. Marrero ${ }^{7}$, C. Rodriguez-Lozano ${ }^{1} .{ }^{1}$ Rheumatology; ${ }^{2}$ Gerontology, Hospital Univ. Gran Canaria Dr. Negrin; ${ }^{3}$ Primary Care, Gerencia Gran Canaria; ${ }^{4}$ Rehabilitation; ${ }^{5}$ Analisis Clinicos; ${ }^{6}$ Orthopedic Surgery, ${ }^{7}$ Radiology, Hospital Univ. Gran Canaria Dr. Negrin, Las Palmas de Gran Canaria, Spain

Background: in March 2012 was implemented a FLS Unit coordinated by Rheumatology

Objectives: communicate the results of the unit in the 2012-2017 period.

Methods: Patients $>50$ years with fragility fracture. The program consists of: 1 ) training of primary care doctors (GP), 2) recruitment from the emergency room or admitted with hip fracture; 3) Bone densitometry; 4) patient education by a nurse; 6) report to GP with recommendations of managing; and 7) follow-up of persistence of treatment (telephonic survey plus prescription in the electronic records) at 3, 6, 12 and 24 months.

Results: The FLS has attended 1739 patients: mean age 73 y, 81\% women. The location of fractures was forearm $(32 \%)$, hip $(24 \%)$, humerus $(21 \%)$, vertebra $(10 \%)$ and other locations (12\%). Previous treatment with bisphosphonate $17 \%$, $10 \%$ of them at baseline.

After the baseline visit, $75 \%$ of patients were sent to GP and $25 \%$ to rheumatology. Treatment with bisphosphonate or equivalent was recommended to 1264 patients $(72 \%)$. Persistence of treatment (analysed at 3, 6, 12 and 24 months in $1,051,823,622$ and 351 patients, respectively) was $74 \%, 72 \%, 75 \%$ and $69 \%$, respectively

\begin{tabular}{lc}
\hline Risk factors (FRAX), $\mathrm{n}(\%)$ & \\
\hline Previous fracture & $323(18)$ \\
Parent's hip fracture & $190(11)$ \\
Glucocorticoids & $148(8)$ \\
Somking & $186(10)$ \\
Alcohol & $68(4)$ \\
Secondary Osteoporosis & $287(16)$ \\
BMl<18.5 & $31(2)$ \\
Rheumatoid arthritis & $40(2)$ \\
Bone Densitometry, $\mathrm{n}(\%)$ & \\
Normal & $159(13)$ \\
Osteopenia & $551(45)$ \\
Osteoporosis & $517(42)$ \\
FRAX, mean (DE) & $13,2(9)$ \\
Major Fracture & $6(7)$ \\
Hip Fracture &
\end{tabular}

Conclusions: Our FLS is effective in terms of beginning and persistence of antifracture treatment in the medium term.

Disclosure of Interest: None declared

DOI: 10.1136/annrheumdis-2018-eular.2361

\section{THU0481 \\ PHYSICAL PERFORMANCE FACTORS INFLUENCING GAIT SPEED IN PATIENTS SURGICALLY TREATED FOR OSTEOPOROTIC HIP FRACTURES}

B.R. Kim. JEJU NATIONAL UNIVERSITY HOSPITAL, Jeju, Korea, Republic of Ireland

Objectives: This study was undertaken to determine physical performance factors associative of gait speed in patients surgically treated for hip fractures.
Methods: Fifty eight patients (16 males and 42 females; average age 79.1 \pm 9.1 years) who underwent a hip surgery due to hip fractures participated in this study. Patients completed 10 metre walk test (10MWT) to assess gait speed. Additional physical performance tests included Timed up and go test (TUG), Berg balance scale (BBS), one repetitive maximum (1RM) of leg extension, leg curl, hip abduc tion of surgical and nonsurgical sides, and instrumental gait analysis for spatiotemporal parameters.

Results: In the bivariate analyses, postoperative 10WMT had a significant positive correlation with the postoperative TUG $(r=0.85, p<0.01)$, age $(r=0.57$, $p<0.01)$, swing phase duration $(r=0.35, p<0.01)$, gait cycle duration $(r=0.49$, $p<0.01)$ and significant negative correlation with the postoperative BBS $(r=-0.69$, $p<0.01), 1 R M$ of surgical leg extension ( $r=-0.35, p=0.01), 1 R M$ of nonsurgical leg extension ( $r=-0.40, p<0.01), 1 \mathrm{RM}$ of surgical leg curl $(r=-0.44, p<0.01), 1 \mathrm{RM}$ of nonsurgical leg curl $(r=-0.41, p<0.01)$, $1 \mathrm{RM}$ of hip abduction $(r=-0.32, p=0.02)$, cadence $(r=-0.53, p<0.01)$, stance phase duration $(r=-0.26, p=0.04)$. In addition, a presence of dementia was significantly correlated with 10WMT (44.2 s vs $22.4 \mathrm{~s}, \mathrm{p}=0.02)$. In the linear regression analyses, the postoperative TUG $(\beta=0.85, p<0.01)$ was a factor associative of the postoperative 10MWT.

Conclusions: This study revealed that the presence of dementia, the postoperative balance ability, muscle strength of surgical and nonsurgical legs were significantly associated with postoperative gait speed 1 month after hip surgery due to hip fractures. Therefore, these results could be importance in planning various postoperative rehabilitative programs to improve gait speed early after hip surgery due to hip fractures.

Disclosure of Interest: None declared

DOI: 10.1136/annrheumdis-2018-eular.1219

\section{THU0482 RELATIONSHIP BETWEEN MILD VERTEBRAL BODY DEFORMITY AND KELLGREN-LAWRENCE'S OSTEOARTHRITIS LEVEL WITH THE PREVALENCE OF NON-TRAUMATIC DORSAL AND LUMBAR BACK PAIN IN FEMALE PATIENTS WITH RISK OF OSTEOPOROTIC VERTEBRAL COLLAPSE}

C.A. Guillen-Astete, C. ljoán, J.R. Quiñones-Torres, M. Vázquez Díaz. Rheumatology Department, Ramon y Cajal University Hospital, Madrid, Spain

Background: There is a lack of information about the meaning of mild vertebral body deformities and its relationship with back pain. Many patients with this kind of wedges are sent to rheumatology clinics to assess the possibility to start treatment for secondary prevention of osteoporosis fractures.

Objectives: The present study aims to determine the relationship of the number of medical consultations due to dorsal or lumbar pain and two categorical variables: Presence or absence of mild vertebral wedge (Genant's first level of classification) and Kellgren-Lawrence's osteoarthritis classification levels I-II and III-IV

Methods: We conducted a retrospective follow-up of 1131 patients with and without mild vertebral body deformities along three years to compare the frequency of axial pain episodes assessed in emergency units and their chance to evolve to moderate or severe wedges.

Results: : In the group without deformities, the cumulative incidence of dorsal or lumbar pain episodes was $7.2 \%$ per year, and the incidence density was 7805 cases per 100 patients-year. In the group with mild deformities, the cumulative incidence of dorsal or lumbar pain episodes was $7.0 \%$ per year, and the incidence density was 7318 cases per 100 patient-year ( $p=0.77$ and 0.58 , respectively). We grouped patients according to their osteoarthritis severity. The cumulative incidence of dorsal and lumbar pain episodes along the period of observation, in patients with a spinal Kellgren-Lawrence's osteoarthritis degree I-II and III-IV were $19.8 \%$ (Cl95\% 12.10\%>27.49\%) and 31.5\% (CI95\% 18.27\%>44.72\%), respectively $(p<0.0001)$. The density of incidence for both groups was 6.18 cases per 100 patients-year and 11.3 cases per 100 patients-year, respectively $(p<0.0001)$. The difference in proportions of patients who developed a moderate or severe Genant's vertebral deformities was not statistically significant among patients with or without mild vertebral wedges.

Conclusions: Our results point that back pain incidence is not related to the presence of mild vertebral wedges but the severity of axial osteoarthritis. Also, the behaviour of starting a secondary osteoporosis prevention treatment after the detection of a mild vertebral wedge is not supported by our results.

Disclosure of Interest: None declared

DOI: 10.1136/annrheumdis-2018-eular.7333 